\title{
Experiencia de pasantía transferida a comunidad pesquera en Costa Rica
}

\author{
Fiorella GonzÁlez SolóRZAno \\ (Encargada de Cátedra Tecnología Agroindustrial) y Karol Saravia Zúñiga (Encargada de Cátedra gerencia y desa- \\ rrollo agroindustrial).Universidad Estatal a Distancia. figonzalez@uned.ac.cr, ksaravia@uned.ac.cr
}

Recibido: 04 de junio de 2018

Aceptado: 10 de agosto de 2018

\section{RESUMEN}

Se transfirió la experiencia de una pasantía educativa universitaria en la universidad autónoma de Nayarit México como parte de la actualización curricular de dos cátedras del Programa Ingeniería Agroindustrial de la Universidad Estatal a Distancia.

A partir de lo observado en visitas guiadas y en capacitaciones recibidas se generó un proyecto de extensión, investigación y docencia, que involucra una comunidad pesquera potencial con necesidades socioeconómicas para transferir una metodología que involucre el manejo del cultivo de un molusco, con la necesidad de mejorar la práctica diaria, dándoles acompañamiento, capacitación y generándoles responsabilidad como parte de solución integral.

Palabras claves: Capacitación, molusco, pasantía, transferencia, comunidad.

\begin{abstract}
The experience of a university educational internship was transferred in the autonomous university of Nayarit Mexico as part of the curricular update of two chairs of the Agroindustrial Engineering Program of the State University at a Distance.

From what was observed in guided visits and training received, an extension and training project was generated that involves a potential fishing community with socioeconomic needs to transfer a methodology that involves the management of a mollusc culture, with the need to improve the practice daily, giving them accompaniment, training and generating responsibility as part of an integral solution.
\end{abstract}

Key words: Training, mollusc, internship, transfer, community.

\section{Introducción}

Como parte de una pasantía a las instalaciones de la Universidad Autónoma de Nayarit (UAN) en México, se visitaron diferentes áreas, facultades y departamentos con la finalidad de visualizar y compartir experiencia con profesionales en áreas afines a la Ingeniería Agroindustrial como: el recurso humano para docencia, plan de estudios, metodología, evaluación de los aprendizajes entre otros.

A partir de esta experiencia, tuvo como objetivo dar a conocer el intercambio de ideas entre docentes y expertos a la visualización y transferencia de conocimiento de diferentes programas y facultades, sin dejar de lado el área investigativa y trabajo de extensión que realiza la UAN. Dentro de los objetivos específicos se tiene relacionar si en ambientes y situaciones similares a la de la pasantía, se pueden generar proyectos con acompañamiento y capacitación a diferentes comunidades. Además, determinar si lo vivido en la pasantía puede generar un acompañamiento y capacitaciones de mejora a una comunidad; determinar la posibilidad de vinculaciones para generar un proyecto luego de la pasantía y determinar la aplicabilidad de la posibilidad de transferencia de metodologías según necesidad de comunidades.

Lo anterior para recopilar y elaborar una síntesis de la información que pudiera ser aplicada y valorada para mejoras e integración a nivel de programa y cátedras del programa Ingeniería Agroindustrial, que pueden transferir en sus asignaturas o a nivel de extensión en una comunidad que lo solicite. 

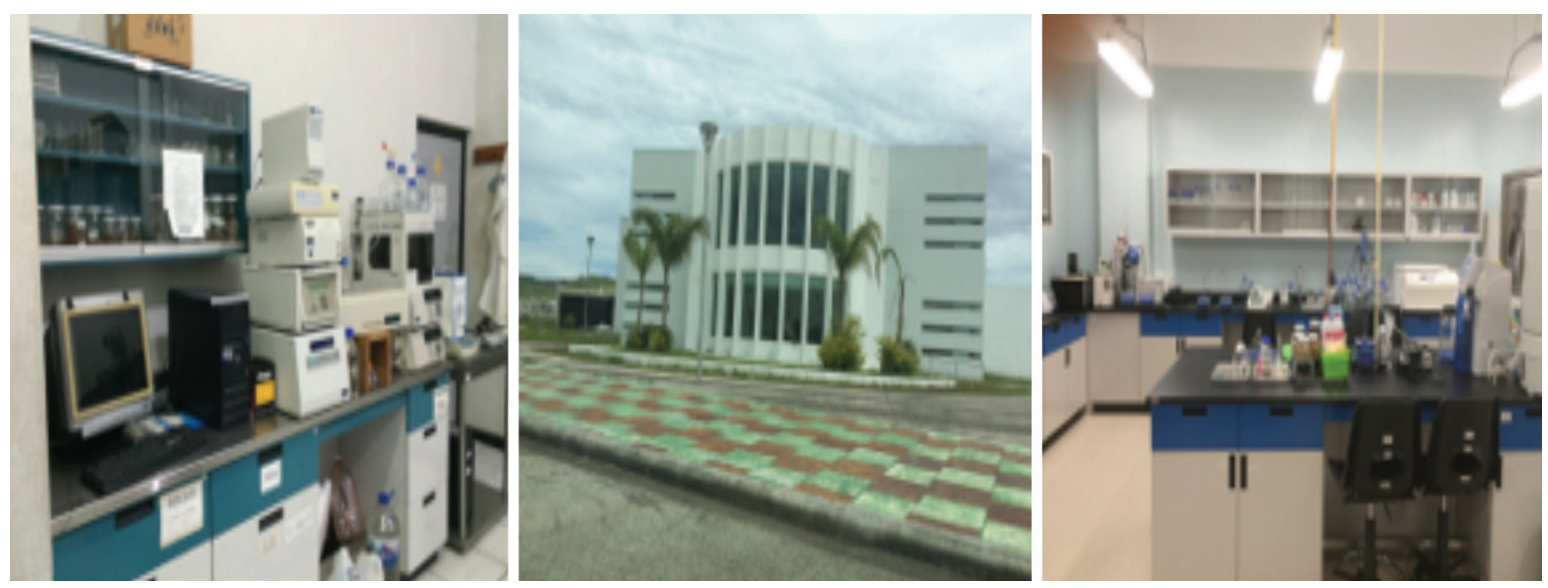

Figura 1. Instalaciones CENIT 2 y laboratorios. Fuente: Propia, (2017).

La Universidad Autónoma de Nayarit es una institución de educación superior con la responsabilidad asignada de formar profesionalmente a la juventud, generar conocimiento vinculado al desarrollo social y difundir la cultura humanista, las manifestaciones artísticas y las innovaciones tecnológicas y científicas (UAN, 2011).

Esta universidad cuenta con redes de colaboración a nivel de carreras en todo el país y a nivel de laboratorios y centros de investigación, además de realizar extensión a diferentes comunidades, en la que participan los docentes, estudiantes y especialistas investigadores.

La Universidad Autónoma de Nayarit, cuenta con una amplia gama de oferta académica en diferentes áreas de especialidad, en el caso que compete es la facultad de ingeniería pesquera la cual está ubicada en San Blas, Nayarit México, tiene la misión de formar profesionales de la pesca, con habilidades y capacidades para la producción de alimentos, con una actitud responsable frente a la explotación de los recursos pesqueros (UAN, 2011).

A partir de esta experiencia se ideó aplicar lo aprendido durante la pasantía en la ejecución de un proyecto en una comunidad pesquera de Costa Rica, con el objetivo de gestionar y dar acompañamiento para impulsar el desarrollo de una metodología que mejore la actividad productiva de las familias involucradas.

\section{Desarrollo}

Durante la pasantía se realizó una visita al Centro Nayarita de Innovación y Transferencia de Tecnología (CENITT 2), (Figura 1) lugar donde se encontraban laboratorios, estaciones de trabajo para los investigadores y principiantes de investigación en estos últimos trabajan estudiantes avanzados de las carreras relacionadas con cada una de las especialidades a obtener el grado de investigador avanzado para el desarrollo de proyectos propios de la institución o relacionados con ayuda o requerimientos de comunidades.

Según la literatura para ejecutar una pasantía se debe contar con un marco teórico que permite una planificación y aplicación que favorece su efectividad (Fullan y Stiegelbauer, 1997; Núñez, 2000). Además, se establece que los pasantes pueden poner a prueba elementos aprendidos en la Pasantía, en su clase cotidiana. Entre los elementos fundamentales, se considera lo siguiente: Aprendizaje por observación y en la práctica. Los profesores participan de estas actividades como un alumno más, pues según Levine (2002), no se puede aprender si no hay práctica. Ambos aspectos son cruciales, pues los perfeccionamientos exitosos acompañan la teoría con demostración, práctica, retroalimentación y entrenamiento (Jungsatikul, 1991 citado en Arancibia, 1994). 
Núñez (2000) indica que los perfeccionamientos no cuentan con modelos teóricos explícitos y evaluados. Falta conocimiento específico sobre los profesores y su práctica, y los estándares que deben alcanzar (Alliaud y Duschatzky, 1998; Sternberg y Horvarth, 1995 citados en Jones, 1997).

En esta visita se recibió la charla de Nuevas tecnologías en acuicultura para el cultivo de camarón y tilapia, de donde surge la idea para ser utilizada como modelo a seguir en el Programa de Ingeniería Agroindustrial en la ejecución de proyectos de investigación y extensión con colaboración de las diferentes cátedras del Programa.

El personal del Centro Nayarita de Innovación y Transferencia de Tecnología lo que realiza es capacitaciones y acompañamiento a nivel de cultivo y comercialización, venden servicios, transfieren metodología, dan acompañamiento, con la finalidad de realizar emprendedurismo y se generen proyectos innovadores y exitosos, lo han realizado como una alternativa guiada para mejorar el trabajo y sustentabilidad de algunas comunidades.
En la UAN se recibió la capacitación sobre el proyecto del camarón blanco que lo realizan en el sureste mexicano, principalmente en Isla Mujeres, el cual tiene como finalidad de transferir estrategia de desarrollo en el cultivo para que no dañen los arrecifes, manglares y aprenda la comunidad a cultivarlo de manera programada y estructurada.

Los investigadores e involucrados en el proyecto, doctores funcionarios de la institución con su debida especialidad, gestionaron este proyecto nacional de innovación y emprendedurismo el cual involucra acompañamiento y capacitación a la comunidad necesitada de iniciativas innovadoras que les ayuden a avanzar y tener un ingreso adicional para las familias involucradas.

Los investigadores se desplazan a la isla mujeres con el paquete tecnológico que incluye diferentes capacitaciones que involucra generalidades y técnicas idóneas propias al cultivo de la especie elegida de camarón. Los investigadores de la UAN aportan además de la técnica, materiales y propuestas acordes a la comunidad a la que se desea transferir (Figura 2).
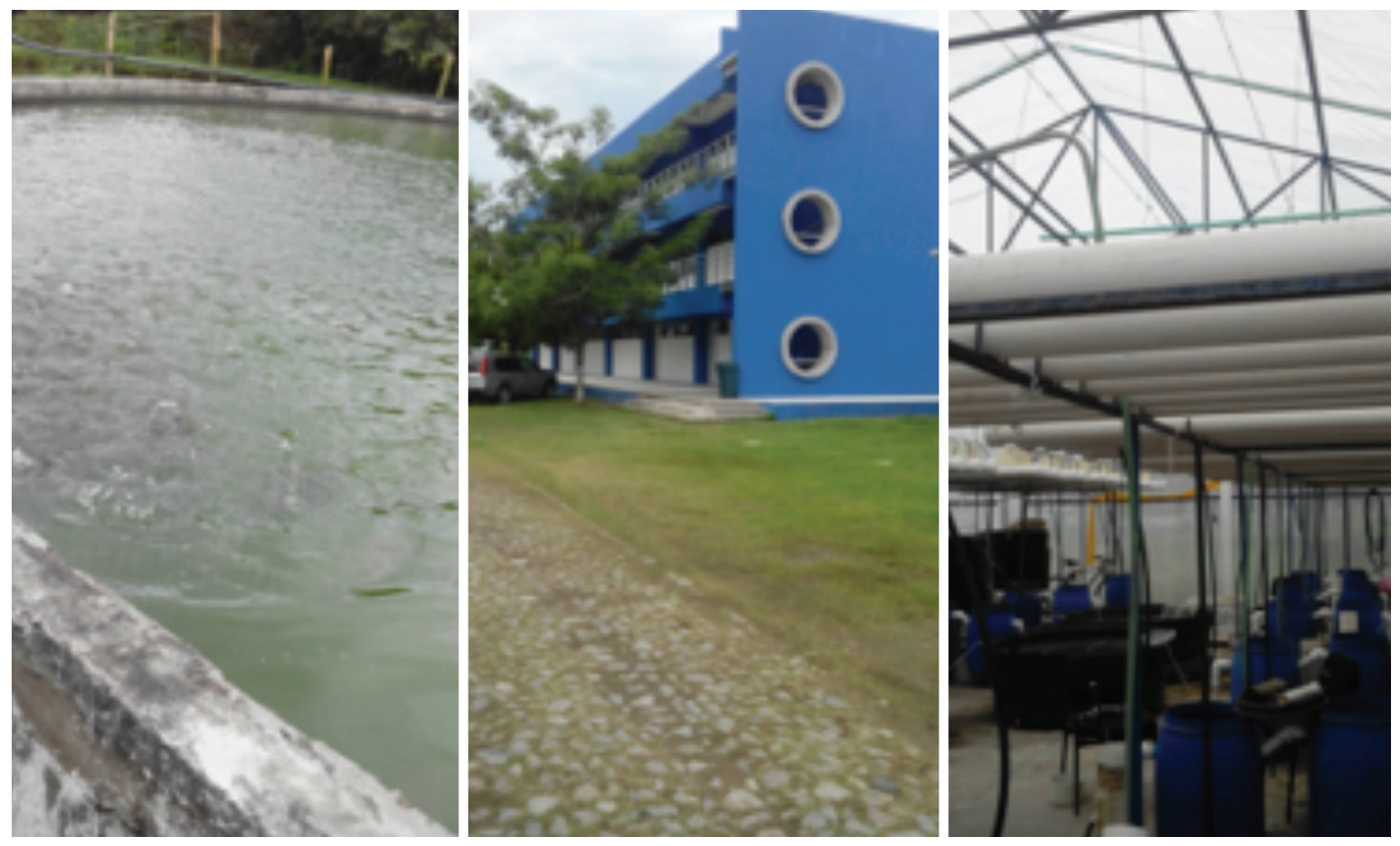

Figura 2. Instalaciones y laboratorios Ingeniería pesquera. Fuente: Propia (2017). 
Este es un trabajo en conjunto con la finalidad de impulsar desarrollo en comunidades atrasadas, con bajos desarrollo socioeconómico, donde los actores (investigadores especialistas, comunidad, municipios, empresas, entre otros) planifican, desarrollan e irán ajustando la técnica y metodología, según diversos factores propios de la isla como: clima, contexto social, cultural económico, con la finalidad de contextualizar para lograr con éxito el rendimiento esperado de la gestión según el prototipo desarrollado en el CENNIT (Centro Nayarita de Innovación y Transferencia de Tecnología) y la teoría que lo involucra.

Según lo indicado anteriormente y con la propuesta existente de un proyecto innovador que involucra a una comunidad con necesidad de apoyo y acompañamiento se buscó trabajar en conjunto con diferentes departamentos en la Universidad Estatal a Distancia para transferir parte de la oportuna experiencia en la UAN.

Se calendarizaron las visitas a la comunidad pesquera elegida por sus condiciones socioeconómicas de previo estudio de otros proyectos que se encuentran en la provincia de Puntarenas (lugar de desarrollo del proyecto) y que están ligados a la municipalidad de la localidad, con la finalidad de capacitarlos con talleres de manipulación de mejillón, biología de bivalvos y darles acompañamiento para poder ofrecerles un mejoramiento socioeconómico comunal de la zona.

En las visitas se planificaron diferentes actividades como: un taller diagnóstico para conocer la realidad de las comunidades cercanas y de la comunidad elegida, situación actual de trabajo, estudio, comercio, ambientales, entre otras.

Dentro de las actividades desarrolladas por los integrantes de la comunidad es la captura no controlada del mejillón o chora en bancos naturales. Por desconocimiento se ha venido destruyendo el banco natural para obtención de la especie y otros similares, que en años pasados se extraían, pero en la actualizad ya desaparecieron como es el caso de la chucheca, por lo que el gobierno del país en complicidad con otras instituciones apoya la iniciativa de capacitar y transferir a la comunidad elegida, una metodología tropicalizada a la y menos invasiva y destructiva que puedan tener como proyecto alternativo a la pesca principalmente en tiempo de veda, donde sean familias que se involucren y encarguen de todo el proceso, desde siembra hasta comercialización; pues conllevaría resultados positivos, no solo económicos sino sociales además de bajar el riesgo en que incurren (venta y consumo de drogas, delincuencia entre otros) por el ocio y pobreza de la zona.

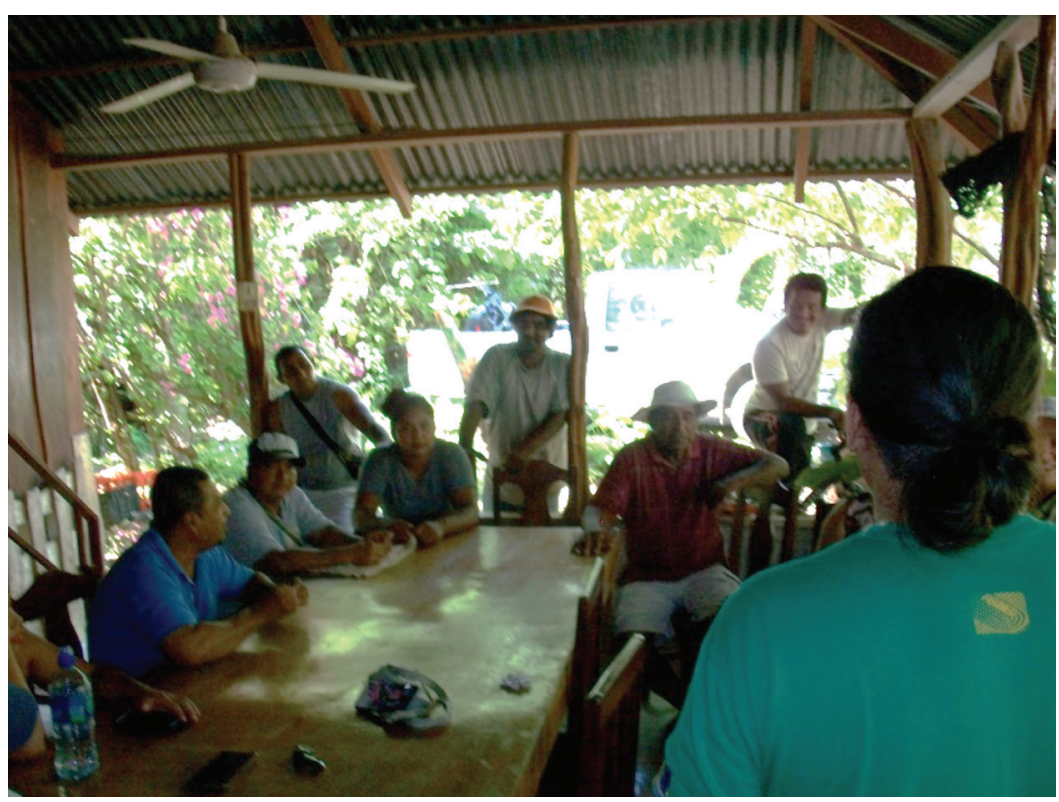

Figura 3. Capacitación introductoria a la comunidad. Fuente: Propia (2017). 
En las primeras visitas a la comunidad se realizaron capacitaciones básicas de manipulación (Figura 3 ) de alimentos y posibles enfermedades a transmitir por este tipo de molusco como lo son hepatitis A, problemas gastrointestinales entre otras, al extraerlo, así como al consumirlo, aunado a la incorrecta manipulación por desconocimiento de los involucrados.

Se les recalcó sobre la responsabilidad que tienen los llamados "Choreros" (personas que extraen el molusco) a la hora de consumirlos en su hogar o venderlos sin ninguna técnica de depuración de por medio, tomando en cuenta el desconocimiento de cómo se alimenta un bivalvo al ser este filtrador y concentrador como tal, además de no tener la conciencia de las condiciones de lavado y desinfectado en cuanto a procedimiento, concentraciones y demás.

En la actualidad se trabaja de forma colaborativa con la comunidad principalmente la asociación de pescadores tanto hombres como mujeres que se reparten algunas tareas como lo son obtención de semilla, cuido, limpieza y están en comunicación entre ellos para coordinar otras posibles tareas relacionadas a la nueva actividad innovación y emprendedurismo.

Con el objetivo de impulsar con responsabilidad social a esta comunidad es que los investigadores una vez al mes enseñan una metodología guiada de como limpiar, mantener vivos los moluscos, técnica de siembra, que es parte de la transferencia de conocimiento y acompañamiento que se le ofrece a los involucrados; donde se pretende tropicalizar una técnica que les ayude desarrollar esta actividad opcional para la actividad de siembra, cosecha y comercialización del molusco dentro y fuera de la zona.

\section{Conclusiones}

Con la experiencia vivida en la pasantía se concluye que en ambientes y situaciones similares se pueden generar proyectos con acompañamiento y capacitación a comunidades con necesidades socioeconómicas que aporten una solución integral.

La experiencia de la pasantía generó una gestión tangible de acompañamiento en la comunidad pesquera seleccionada logrando capacitar en temas básicos como manipulación de alimentos gracias a la apertura, disponibilidad y necesidad de la comunidad. La experiencia generó la ejecución de un proyecto con un acompañamiento en una comunidad pesquera con necesidad de una actividad alternativa que la involucre y genere una mejora socioeconómica.

Se puede generar vinculación con diferentes áreas de la universidad visitada (laboratorios, departamento de movilidad estudiantil, investigadores) que pueda apoyar a los posibles nuevos proyectos a generarse.

Finalmente, esta experiencia da la posibilidad de lograr una aplicabilidad en condiciones reales y que permita transferir metodologías a los involucrados de la comunidad, que tengan alguna carencia subsanable donde se debe involucrar y vincular la extensión guiada y capacitación estructurada para llenar una necesidad.

\section{Referencias bibliográficas}

Alliaud, A. \& Duschatzky, L. (Comps.). (1998). Maestros. Formación práctica y transformación escolar. Buenos Aires: Miño y Dávila Editores.

Jones, M. (1997). Trained and untrained secondary school teachers in Barbados: Is there a difference in classroom performance? Educational Research, 39(2), 175-184

Arancibia, V. (1994). Formación y capacitación de los profesores: Impacto en el aprendizaje de los Estados Unidos. Proyecto Principal de Educación, 34, 51-76. Santiago: UNESCO/OREALC.

Arancibia, V. \& García, C. (2003). Diseño de un sistema de pasantías PENTA UC. Santiago: Proyecto FONDEF DO2I1039.

Arancibia, V. \& García, C. (2007). Pasantías PENTA UC: Una Propuesta Innovadora de Desarrollo Profesional Docente. Psykhe v.16 n.1: Santiago. Recuperado de http://dx.doi.org/10.4067/ S0718-22282007000100011

Arancibia, V., Herrera, P. \& Strasser, K. (1999). Psicología de la educación. México D.F.: Alfaomega.

Fullan, M. \& Stiegelbauer, S. (1997). El cambio educativo. México, D.F: Trillas. 
Levine, D. (2002). Thinking about doing. On learning from experience and the flight from thinking. Human Relations, 55(10), 1251-1268.

Núñez, I. (2000). Los docentes y la política de desarrollo profesional. Recuperado desde www.mineduc.cl
Universidad autónoma de Nayarit (2011) Unidad académica escuela de ingeniería pesquera recuperado de: http://www.uan.edu.mx/unidad-academica-escuelanacional-de-ingenieria-pesquera

Universidad autónoma de Nayarit (2011) Perfil Universidad Autónoma de Nayarit recuperado de: http://www. asuntosinternacionalesuan.com/uan/perfil/ 